\title{
Práticas de educação em saúde desenvolvidas pelo núcleo de ensino e pesquisa (NEP) de um hospital do interior do estado do Pará
}

\author{
Health education practices developed by the teaching and research center (TRC) of a \\ hospital in the interior of the state of Pará
}

\begin{abstract}
Prácticas de educación para la salud desarrolladas por el centro de enseñanza e investigación (CEI) de un hospital en el interior del estado do Pará
\end{abstract}

\begin{abstract}
Herberth Rick dos Santos Silva ${ }^{1 *}$, José Benedito dos Santos Batista Neto ${ }^{1}$, Benedito do Carmo Gomes Cantão ${ }^{1}$, Anderson Bentes de Lima ${ }^{1}$, Jaylen França Cunha1, Ailson Almeida Veloso Júnior $^{1}$, Jackson Luís Ferreira Cantão ${ }^{1}$, Caroline Lima Garcia ${ }^{1}$, Amanda Ouriques de Gouveia ${ }^{2}$, Genislaine Ferreira Pereira ${ }^{3}$.
\end{abstract}

\section{RESUMO}

Objetivo: Relatar a experiência de estagiários do Núcleo de Ensino e Pesquisa (NEP) na aplicabilidade de práticas de educação permanente em pacientes e profissionais e seu consequente êxito, em um hospital de médio porte do interior do Pará. Relato de Experiência: Trata-se de um estudo do tipo relato de experiência, feito por estagiários do NEP, realizado após 9 meses de atuação em um hospital localizado no sudeste paraense. Entre os objetivos e atividades realizadas no NEP, estão: o estímulo do trabalho em equipe egestão em saúde, a organização de eventos de cunho educacional dentro do hospital, a organização de cursosde capacitação e aperfeiçoamento profissional para internos já atuantes, a promoção de ações beneficentese de saúde, além da organização e controle de estagiários advindos de instituições de Ensino, tendo em vista o caráter de hospital-escola, tido pela instituição. Considerações Finais: Todas essas práticas, tiveram como objetivo melhorar o quadro da saúde coletiva e preventiva no munícipio, munidas também de ensino e experiência aos estagiários, que a partir da execução de tais metodologias ativas, desenvolveram práticas de gerenciamento e de educação em saúde, demonstrando e efetivando assim a eficácia de tais métodos de ensino.

Palavras-chave: Educação em saúde, Promoção da saúde, Educação em enfermagem.

\begin{abstract}
Objective: To report the experience of interns at the Teaching and Research Center (TRC) in the applicability of permanent education practices to patients and professionals and their consequent success, in a medium-sized hospital in the interior of Pará. Experience report: This is an experience report type study, carried out by TRC trainees, carried out after 9 months of experience in a hospital located in southeastern Pará. Among the objectives and activities carried out at the TRC, are: the encouragement of teamwork and health management, the organization of educational events within the hospital, the organization of training and professional development courses for already active interns, the promotion of charitable actions health, in addition to the organization and control of trainees from educational institutions, in view of the character of a teaching hospital, held by the institution. Final considerations: All of these practices were aimed at
\end{abstract}

1Universidade do Estado do Pará (UEPA). Tucuruí - Pará.

*E-mail: rick.santos.hr@gmail.com

${ }^{2}$ Fundação Santa Casa de Misericórdia do Pará, Belém - Pará.

3 Universidade Anhanguera (UNIDERP). Tucuruí - Pará. 
improving the collective and preventive health situation in the municipality, also providing the trainees with teaching and experience, who, through the implementation of such active methodologies, developed health management and education practices, demonstrating and carrying out so the effectiveness of such teaching methods.

Key words: Health education, Health promotion, Education in nursing.

\section{RESUMEN}

Objetivo: Informar sobre la experiencia de los pasantes en el Centro de Enseñanza e Investigación (CEI) en la aplicabilidad de las prácticas de educación permanente a pacientes y profesionales y su consecuente éxito, en un hospital de tamaño mediano en el interior de Pará. Informe de experiencia: Este es un estudio tipo informe de experiencia, realizado por aprendices de CEI, realizado después de 9 meses de experiencia en un hospital ubicado en el sureste de Pará. Entre los objetivos y actividades que se llevan a cabo en la CEI se encuentran: el fomento del trabajo en equipo y la gestión de la salud, la organización de eventos educativos dentro del hospital, la organización de cursos de capacitación y desarrollo profesional para pasantes ya activos, la promoción de acciones caritativas de salud, además de la organización y el control de pasantes de instituciones educativas, en vista del carácter de un hospital docente, en poder de la institución. Consideraciones finales: Todas estas prácticas tenían como objetivo mejorar la situación de salud colectiva y preventiva en el municipio, también proporcionar a los alumnos enseñanza y experiencia, quienes, a través de la implementación de tales metodologías activas, desarrollaron prácticas de gestión y educación en salud, demostrando y llevando a cabo entonces la efectividad de tales métodos de enseñanza.

Palabras clave: Educación en salud, Promoción de la salud, Educación en enfermaría.

\section{INTRODUÇÃO}

A formação profissional de qualidade deve possuir uma base sólida de formação geral, que não se finaliza na academia, mas que acontece durante a evolução do ser humano, por meio da educação permanente (PASCHOAL AS, et al., 2006). Nesse âmbito, visando a constante evolução profissional dos trabalhadores de serviços de saúde, assim como o crescimento individual das pessoas que frequentam estes serviços, como hospitais, é fundamental que esses estabelecimentos abriguem um Núcleo de Ensino e Pesquisa (NEP).

A proposta da Educação Permanente em Saúde (EPS), apresentada pelo Ministério da Saúde, torna-se estratégia fundamental para a recomposição das práticas de formação, bem como das práticas pedagógicas e de saúde. Esta, visa a busca por articulações de serviços e gestão setorial, atenção e controle social, tendo em vista a efetiva implementação dos princípios e das diretrizes preconizadas pelo Sistema Único de Saúde (SUS) (BRASIL, 2004).

Um dos motivos que tornam o referido hospital um estabelecimento de excelência é a presença do NEP, haja vista que o mesmo proporciona processos educativos coletivos que visam o desenvolvimento e aperfeiçoamento dos funcionários, a partir de uma série de atividades comumente denominadas de workshops, capacitações e treinamentos.

Para que o NEP funcione corretamente é importante que os integrantes da equipe, formada por profissionais e estagiários do núcleo, possuam um estímulo a gestão, uma vez que a compreensão da organização do trabalho para a produção de serviços de saúde possibilita, nesta perspectiva de reconceitualização, visualizar a totalidade dos problemas, possibilitando a formulação de estratégias de superação pautadas na realidade concreta (LOPES SVLM, et al., 2015).

Logo, percebe-se, que o NEP, não é apenas importante para o seu público alvo, é uma oportunidade de crescimento profissional para seus organizadores e principalmente para os acadêmicos envolvidos no 
projeto, ao passo que, nestes, é estimulado o senso de gestão por meio de metodologias ativas aos quais são submetidos.

As metodologias ativas têm o potencial de despertar interesse, à medida que os alunos se inserem na teorização e trazem elementos novos, ainda não considerados nas aulas ou na própria perspectiva do professor ou outro profissional envolvido. Quando acatadas e analisadas as contribuições dos alunos, valorizando-as, são estimulados os sentimentos de engajamento, percepção de competência e de pertencimento, além da persistência nos estudos (BERBEL NAM, 2011). Portanto, a partir do momento em que os estagiários do NEP são estimulados a executar tarefas de gerenciamento para a solução e promoção de estratégias para o enfrentamento de desafios que venham surgir no ambiente hospitalar, estes, criam autonomia de pensamento para a resolução de tais.

É a partir deste contexto que o presente relato de experiência se desenvolveu. Tal relato busca descrever as experiências de estagiários do NEP do um hospital público do interior do Pará na aplicabilidade da práticas de educação permanente em pacientes e profissionais, bem como relatar a importância de tal Núcleo para a comunidade acadêmica e profissional.

\section{RELATO DE EXPERIÊNCIA}

Este estudo, trata-se de um relato de experiência da equipe de estagiários do Núcleo de Ensino e Pesquisa (NEP) de um hospital localizado em um município do sudeste paraense, instituição de referência no setor de Urgência e Emergência na região Sul e Sudeste do Pará. Os estagiários eram acadêmicos do curso de Enfermagem de uma universidade pública localizada no município em questão, onde, foram selecionados a partir de um processo seletivo em que seriam escolhidos quatro discentes. Tal estágio, sendo, este, extracurricular, tinha como objetivos: o estímulo do trabalho em equipe e gestão em saúde, a organização de eventos de cunho educacional dentro do hospital, a organização de cursos de capacitação e aperfeiçoamento profissional para internos já atuantes, a promoção de ações beneficentes e de saúde, além da organização e controle de estagiários advindos de instituições de Ensino, tendo em vista o caráter de hospital-escola, tido pelo hospital.

Os acadêmicos receberam orientação e treinamento do coordenador do NEP, este, sendo docente da instituição de ensino UEPA e Enfermeiro atuante no hospital, através de metodologias ativas de ensino, que os levaria à prática de atividades de gerenciamento e Educação em Saúde. O programa de estágio extracurricular, gerou aos participantes, ao seu fim, além de conhecimento e práticas em saúde, 230 horas de carga horária, exercidas do dia 8 de maio ao dia 11 de dezembro de 2019. Entre as diversas atividades importantes realizadas pelo NEP, tivera a da conscientização dos profissionais de saúde atuantes no hospital quanto a importância da lavagem das mãos, referente aos critérios de segurança ao paciente.

Com isso, foi-se posto aos estagiários uma experiência de metodologia ativa, por meio do Arco de Maguerez, que de acordo com Reibnitz KS e Prado ML (2006) foi apresentada pela primeira em 1982, sendo que desde então é utilizada como metodologia para a realização de ações de educação para a solução de situações que estejam relacionadas com a vida em sociedade. A experiência foi realizada em 5 etapas, como proposto pela metodologia antes citada, sendo elas:

1) Observação da realidade: foi solicitado a equipe do NEP observar as possíveis causas dos altos índices de infecção no ambiente hospitalar, notificados pelo Centro de Controle de Infecções Hospitalares $(\mathrm{CCIH})$, afim de identificar a causa-problema e propor uma possível solução. Durante investigação em algumas clínicas e conversa com os profissionais, foi-se observado o presente descaso quanto aos critérios de segurança ao paciente, principalmente ao que refere-se à lavagem das mãos. Assim, os acadêmicos seguiram para a segunda fase do processo;

2) Identificação de Pontos-chave: ao observar a realidade, percebeu-se os pontos-chave presentes naquela realidade hospitalar: a sobrecarga de trabalho, o descaso quanto a lavagem de mãos e o esquecimento das práticas de segurança do paciente ensinadas na academia. Portanto, estabeleceram-se metas para a resolução dessa problemática; 
3) Teorização: após pesquisa, em bancos de dados eletrônicos, como o Scientific Eletronic Library Online (SCIELO), percebe-se que muitos profissionais não perpetuam esse hábito por não conseguirem visualizar o efeito e resultado imediato do ato no paciente (MARTINI AC e DALL'AGNOL CM, 2005), em contrapartida é evidente que a infecção cruzada é considerada uma das principais causas das infecções hospitalares, cuja transmissão se faz também através das mãos dos funcionários da área da saúde, logo a prática da lavagem das mãos se torna imprescindível (BRASIL, 1998). Segue-se então a quarta fase do processo;

4) Hipótese de solução: após o estudo científico, chegou-se à conclusão de que os profissionais precisam de educação continuada, pois essa é imprescindível para o crescimento professional e qualidade do serviço dos indivíduos já atuantes, de acordo com o Ministério da Saúde (BRASIL, 2019; BRASIL, 2007.) A educação permanente em saúde apresenta-se como uma proposta de ação estratégica capaz de contribuir para a transformação das práticas de saúde. Com isso, pensou-se na elaboração de uma estratégia que faça esses profissionais se conscientizarem da importância de tal ato, bem como lembrarIhes do que aprenderam na academia.

5) Aplicação à realidade: a partir da determinação da hipótese de solução e o embasamento científico coletado para a elaboração de um projeto para mudar a situação-problema, estabeleceram-se 4 passos a serem aplicados: a realização de uma entrevista individual junto aos membros da equipe de enfermagem do HRT, para ter conhecimento sobre seus conceitos e métodos de lavagem de mãos, como o tempo do processo, o modo de fazê-lo e a importância deste.

A partir da percepção dos principais erros foi elaborado um roteiro de palestras para relembrá-los e conscientizá-los da importância da lavagem das mãos nos critériosde segurança do paciente, além do ensino de músicas para o acompanhamento do tempo correto de lavagem. Após tal elaboração, foi então levado a aplicabilidade junto aos profissionais da equipe de enfermagem, e após tal ação, foi então aplicado um questionário, para assim, chegarmos à conclusão de que as ações de educação em saúde tiveram êxito e foram bem recebidas pelos profissionais.

Também, entre as atividades realizadas pelos estagiários, estava a organização de eventos de capacitação e aperfeiçoamento profissionalreferente aos mais diversos assuntos da prática hospitalar, sendo essas realizadas por profissionais especialistas convidados.

O NEP atua na organização do evento, seleção dos palestrantes, divulgação desses atos de educação continuada, seleção do conteúdo a ser repassado, e organização da cronologia do evento, sendo realizados em diversas datas e turnos para que todos os profissionais atuantes tivessem a chance de participar, além da produção de certificados ao profissionais. Também, levando os estagiários ao êxito na execução e atuação no gerenciamento em enfermagem. Outra forma de atuação, sendo essa uma das mais importantes dentro do serviço de saúde pública, estava na participação e organização de eventos que ressaltavam a importância da prevenção na saúde coletiva.

Como exemplo, deu-se o gratificante evento realizado em ode ao outubro Rosa, mês de prevenção ao câncer de mama e de colo do útero. Os estagiários no Núcleo de Ensino e Pesquisa, junto à direção do hospital, selecionaram inúmeros acadêmicos, capacitados previamente, para a execução da tarefa no hall de entrada do hospital e na parte interna do hospital, para, assim, realizar a conscientização, tanto em pacientes internados, quanto as suas acompanhantes e visitantes.

Foram repassados materiais didáticos, demonstrações práticas do exame de auto toque, tirada de dúvidas, além da distribuição de brindes confeccionados pelos própriosestágiários. O evento teve êxito e assim foi repetido nos demais meses seguintes, com outras temáticas, como a da prevenção ao câncer de próstata, no conhecido como novembro Azul. Todas essas práticas, tiveram como objetivo melhorar o quadro da saúde coletiva e preventiva no munícipio, oferecer educação continuada aos profissionais atuantes, além de munir, também, de ensino e experiência aos estagiários, que a partir da execução de tais tarefas, desenvolveram práticas de gerenciamento e de educação em saúde, demonstrando e efetivando assim a eficácia de tal método de ensino. 


\section{DISCUSSÃO}

A promoção da saúde, como a realizada pelo Núcleo de Ensino e Pesquisa (NEP) do hospital, segundo Araújo MRN e Assunção RS (2004) não constitui responsabilidade restrita do setor saúde, mas de uma integração entre os diversos setores do governo municipal, estadual e federal. Setores este como o da educação, que adjunto a saúde,podem gerar ações que culminem com a melhoria das condições de vida da população e da oferta de serviços essenciais aos seres humanos.

Em um estudo realizado por Lavich CRP et al. (2017) comprova a eficência de núcleos semelhantes ao NEP, aqui descrito, no momento em que afirma que departamentos como esse são capazes de planejar estratégias para resolver impasses em unidades de atentimento com a utilização da educação permamentente em saúde. Sendo assim, é imprescindível a implementação e a perpetuação de setores como esse em outras instituições.

A atribuição dos profissionais de saúde, como agentes de mudança, passa a ser de facilitadores no processo da educação em saúde. Processo esse, tido como objetivo central na estruturação do NEP. Essa perspectiva da necessidade de um processo educacional adjunto ao processo de fazer saúde, converge para a formação continuada dos profissionais de saúde visto que, segundo Ceccim $R B$ e Ferla $A A$ (2003):"uma profunda renovação das organizações de saúde não se faz sem uma política de educação para o setor". Destaca-sea educação em saúde, como estratégia de promoção à saúde no processo de conscientização individual e coletiva de responsabilidades e direitos à saúde, estimulando ações que atendam aos princípios do SUS.

A partir deste enfoque, segundo Catribi AMF (2004) devem-se elegerações didáticas que conduzam uma transformação dos indivíduos, ampliando sua capacidade de compreensão da complexidade dos determinantes de fazer saúde e do ser saudável, ações essas, estratégicamente articuladas, como no contexto de atuação dos estagiários do Núcleo de Ensino e Pesquisa, postos para atuar mediante a metodologias ativas, afim de elencar soluções a problemas encontrados, atuando no contexto da promoção e educação em saúde, promovendo também, práticas de educação permanente.

A Educação Permanente em Saúde comporta-se como uma estratégia capaz de contribuir para a transformação dos processos formativos, das práticas pedagógicas e de saúde, para organização dos serviços, executando um trabalho articulado entre o sistema de saúde, em suas várias esferas de gestão, e as instituições formadoras (BRASIL, 2004; BRASIL, 2007). Referente a atuação gerencial, dentro do NEP, exercída pelos atuantes, discentes e docente, segundo Gaidzinski RR et al. (2004) não há mais como negar a importância da função gerencial como elemento integrante do trabalho do enfermeiro, entendido numa lógica que privilegia os interesses coletivos e dando concretude a uma assistência segura que leve em consideração as reais necessidades da clientele.

Ademais, não Podemos deixar de falar sobre os ganhos pessoais e profissionais dos estagiários atuantes no NEP. Estágios, no geral, são capazes de proporcionar aos discentes de enfermagem o desenvolvimento de técnicas, habilidades e conheciementos, que contribuirão não só para o seu processo de formação acadêmica como também para a vida profissional que lhes aguarda após a formação universitária (RESTELATTO MTR e DALLACOSTA FM, 2018).

Nesse sentido, percebe-se que a efetivação de um setor de Ensino e Pesquisa no Hospital e ainda a participação de acadêmicos como estagiários, neste, só tende a acrescentar em todos os envolvidos. Ao fornecer uma educação continuada aos profissionais atuantes, fortalece-se os preceitos dos cuidados e segurança do paciente, que no dia-a-dia do trabalho, podem cair em esquecimento. Ao fornecer palestras realizadas por estudante, estimula-se assim o conhecimento de ambas as partes, dos palestrantes e dos ouvintes, e isso, também, fará com que esses acadêmicos, como futuro profissionais, deem mais atenção ao assunto que fora explanado. No âmbito do gerenciamento, a organização de eventos, só acrescenta aos organizadores, estimulando o espírito de liderança, que é imprescindível para o profissional enfermeiro, também gerando uma rede de informações ao público alvo dos eventos, como conhecimento preventivo, esse, muitas vezes esquecido na área hospitalar. 
Outrossim, se faz necessária a continuidade do programa, uma vez que os benefícios são dirigidos a ambas partes. Além da oportunidade do acadêmico em estar no ambiente em que irá atuar em alguns anos e da familiarização, traz a necessidade e desejo da busca por uma constante melhora do setor da saúde desde o momento da graduação, instigando a atualização, qualidade e, principalmente, no trabalho ativo, em prol de uma saúde de referência, que atenda com segurança e que estreite a relação pacienteprofissional, visando em um processo intervencionista no que concerne ao processo de saúde e doença e que atenda aos princípios do Sistema Único de Saúde.

Conclui-se então, que o Núcleo de Educação e Pesquisa do referido hospital é efetivo quando analisado do ponto de vista metodológico, no qual por meio da realização de ações de educação em saúde permanete consegue previnir, combater e solucionar adiversidadades que a instituição venha enfrentar, o que permite ao hospital oferecer um bom atendimento em saúde para o município. Ademais, além de trazer ganhos para a instituição que o possui, o NEP permite aos estagiários adquirirem experiências, habilidades e conhecimentos, que poderão ser usados, futuramente, em suas vidas profissionais, garantindo assim, uma troca justa de serviços entre as partes envolvidas.

\section{REFERÊNCIAS}

1. ARAÚJO MRN, ASSUNÇÃO RS. A atuação do agente comunitário de saúde na promoção da saúde e na prevenção de doenças. Revista Brasileira de Enfermagem, 2004; 57(1): 19-25.

2. BRASIL. Ministério da Saúde. Portaria oํ 1996/GM/MS, de 20 de agosto de 200: diretrizes para a implementação da política nacional de educação Permanente em Saúde. Brasília, 2007.

3. BRASIL, Ministério da Saúde. Secretaria Nacional de Organização e Desenvolvimento de Serviços de Saúde. Programa de Controle de Infecção Hospitalar. Lavar as Mãos: Informação para Profissionais de Saúde. Brasília: Ministério da Saúde, 1989.

4. BRASIL. Ministério da Saúde. Secretaria de Gestão do Trabalho e da Educação na Saúde. Departamento de Gestão da Educação na Saúde. Política de educação e desenvolvimento para o SUS. Brasília, 2004.

5. BERBEL NAN. As metodologias ativas e a promoção da autonomia de estudantes. Semina: Ciências Sociais e Humanas, 2011; 32(1): 25-40.

6. CATRIB AMF, et al. Promoção da Saúde: saber fazer em construção. In: Barroso GT, Vieira NFC, Varela ZMV, organizadores. Educação em saúde: no contexto da promoção humana. Fortaleza: Demócrito Rocha, 2003; p. 31-8.

7. CECCIM RB, FERLA AA. Residência integrada em saúde: uma resposta da formação e desenvolvimento profissional para a montagem do projeto de integralidade da atenção à saúde. In: Construção da integralidade: cotidiano, saberes e práticas em saúde. 2003. p. 211-226.

8. GAIDZINSKI RR, et al. Liderança: aprendizado contínuo no gerenciamento em enfermagem. Revista Brasileira de Enfermagem, 2004; 57(4): 464-466.

9. LAVICH CRP, et al. Ações de educação permanente dos enfermeiros facilitadores de um núcleo de educação em enfermagem. Revista Gaúcha de Enfermagem, 2017; 38(1): e62261.

10. LOPES SVLM, et al. Relato de experiência: implantação do núcleo de educação permanente em JataúbaPE. Revista da Universidade Vale do Rio Verde, 2015; 13(1):165-174.

11. MARTINI AC, DALL'AGNOL CM. Por que lavar ou não as mãos?: motivos de um grupo de enfermagem. Revista Gaúcha de Enfermagem, 2005; 26(1): 88-101.

12. PASCHOAL AS, et al. A educação permanente em enfermagem: subsídios para a prática profissional. Revista Gaúcha de Enfermagem, 2006; 27(3):336-343.

13. REIBNITZ KS, PRADO ML. Inovação e educação em enfermagem. Florianópolis: Cidade Futura, 2006; 240p.

14. RESTELATTO MTR, DALLACOSTA FM. Vivências do acadêmico de enfermagem durante o estágio com supervisão indireta. Revista Enfermagem em Foco, 2018; 9(4): 34-38. 